\title{
Microverme da aveia como estratégia alimentar na larvicultura do Betta splendens
}

\author{
Oat microverm as food strategy in Betta splendens larviculture \\ Microorganismos de avena como estrategia de alimentación en larvicultura de Betta splendens
}

Recebido: 08/12/2021 | Revisado: 13/12/2021 | Aceito: 19/12/2021 | Publicado: 03/01/2022

Veruska Dilyanne Silva Gomes
ORCID: https://orcid.org/0000-0002-4169-2491
Enstituto Federal de Educação, Ciência e Tecnologia do Tocantins, Brasil
E-mail: veruska_sgomes@yahoo.com.br
Alda Lúcia de Lima Amâncio
ORCID: https://orcid.org/0000-0003-1312-6537
Universidade Federal da Paraíba, Brasil
E-mail: alda.amancio@ yahoo.com.br
Cácio Ribeiro Cavalcanti
ORCID: https://orcid.org/0000-0002-3373-8904
Universidade Federal de Campina Grande, Brasil
E-mail: ksio83@hotmail.com
Matheus Henrique Cardoso de Araújo
ORCID: https://orcid.org/0000-0003-1320-8479
Universidade Federal da Paraíba, Brasil
E-mail: mhca.matheus@gmail.com
Viviane da Silva Medeiros
ORCID: https://orcid.org/0000-0002-8299-9529
Universidade Federal do Rio Grande do Norte, Brasil
E-mail: vivianemedeiros.eaj@gmail.com
João Marcos Monteiro Batista
ORCID: https://orcid.org/0000-0001-9759-093X
Universidade Federal de Goiás, Brasil
E-mail: joao_92trimonteiro@hotmail.com
Nágela Maria Henrique Mascarenhas
ORCID: https://orcid.org/0000-0001-9059-3695
Universidade Federal de Campina Grande, Brasil
E-mail: eng.nagelamaria@gmail.com

\section{Resumo}

A larvicultura é um período crítico na produção de peixes ornamentais, devido às necessidades produtivas e nutricionais das larvas nos primeiros dias de vida. O fornecimento de alimento vivo é essencial para a sobrevivência das larvas, sendo a Artemia sp. o alimento mais utilizando, apensar de representar custo elevado. Tornando necessária a busca por alimentos vivos de menor custo como o nematóide microverme da aveia. O presente trabalho foi desenvolvido com o objetivo de avaliar, por meio de índices de crescimento, desenvolvimento e sobrevivência, o fornecimento do microverme de forma exclusiva ou associado a artêmia e plâncton para larvas Betta splendens. As larvas alimentadas com microverme e artêmia apresentaram maior peso final, comprimento final, ganho em comprimento, maior taxa de desenvolvimento específico e sobrevivência. A taxa de uniformidade do lote no tratamento que recebeu apenas microverme não diferiu dos demais tratamentos, no entanto, as larvas submetidas ao regime alimentar com microverme e artêmia apresentaram melhor taxa de uniformidade quando comparas as que foram alimentadas com microverme e plâncton. A estratégia alimentar visando fornecer microverme e artêmia de forma conjunta promoveu os melhores índices de crescimento, desenvolvimento e sobrevivência na larvicultura inicial do peixe ornamental Betta splendens.

Palavras-chave: Artemia sp.; Nutrição de larvas; Panagrellus redivivus; Piscicultura ornamental.

\begin{abstract}
Larviculture is a critical period in the production of ornamental fish, due to the productive and nutritional needs of the larvae in the first days of life. The supply of live food is essential for the survival of the larvae, being Artemia sp. the most used food, although it represents a high cost. Making it necessary to search for lower cost live foods such as the nematode microworm. The present work was developed with the objective of evaluating, by means of growth, development and survival indices, the microverminal supply exclusively or associated to artemia and plankton for Betta splendens larvae. Larvae fed with microworm and artemia showed higher final weight, final length, length gain, higher rate of specific development and survival. The rate of uniformity in the treatment that received only microverm did not differ from the other treatments, however, the larvae submitted to the microworm and artemia diet showed a better uniformity rate when compared to those fed with microverm and plankton. The feeding strategy aimed at supplying
\end{abstract}


microverm and artemia together promoted the best growth, development and survival rates in the initial Betta splendens larviculture.

Keywords: Artemia sp.; Larvae nutrition; Panagrellus redivivus; Ornamental fish.

\section{Resumen}

La larvicultura es un período crítico en la producción de peces ornamentales, debido a las necesidades productivas y nutricionales de las larvas en los primeros días de vida. El suministro de alimento vivo es fundamental para la supervivencia de las larvas y Artemia sp. el alimento más utilizado, aunque representa un alto costo. Haciendo necesaria la búsqueda de alimentos vivos de menor costo como el nematodo del microworm de la avena. El presente trabajo se desarrolló con el objetivo de evaluar, a través de índices de crecimiento, desarrollo y supervivencia, el aporte del microworm exclusivo o asociado al camarón de salmuera y plancton para larvas de Betta splendens. Las larvas alimentadas con microworm y camarones de salmuera tuvieron mayor peso final, longitud final, ganancia de longitud, mayor tasa de desarrollo específico y supervivencia. La tasa de uniformidad del lote en el tratamiento que recibió solo microworm no difirió de los otros tratamientos, sin embargo, las larvas alimentadas con microworm y camarón de salmuera mostraron una mejor tasa de uniformidad en comparación con aquellas alimentadas con microworm y plancton. La estrategia de alimentación con el objetivo de suministrar microworm y camarón de salmuera juntos promovió las mejores tasas de crecimiento, desarrollo y supervivencia en la larvicultura inicial de los peces ornamentales Betta splendens.

Palabras clave: Artemia sp.; Nutrición larvária; Panagrellus redivivus; Piscicultura ornamental.

\section{Introdução}

O peixe Betta splendens é uma das espécies ornamentais mais comercializadas mundialmente. Os exemplares disponíveis atualmente no mercado são produto de cruzamentos realizados, ao longo dos anos, a partir de espécies selvagens que possibilitaram o desenvolvimento de linhagens com cores, tamanhos e nadadeiras variadas (Ramos \& Gonçalves, 2019). A espécie apresenta características desejáveis como suas cores intensas, respiração aérea não requerendo aeradores nos aquários (Zuanon et al., 2009) e fácil reprodução em cativeiro, utilizando um aquário pequeno e substrato na superfície da água para que o macho construa o ninho de bolhas (Srikrishnan et al., 2017). No entanto, existem dificuldades durante a fase de larvicultura que refletem na redução da taxa de sobrevivência e desempenho zootécnico dos indivíduos nos primeiros dias de vida.

Aumentar a eficiência produtiva na fase de larvicultura é um dos principais fatores que contribuem para a sobrevivência dos peixes Betta splendens em sistema de produção de peixes ornamentais (Fosse et al., 2013). Nesse âmbito, estudos vêm sendo desenvolvidos com o intuito de definir condições ambientais, nutricionais e protocolos de manejo que promovam o aumento dos índices produtivos. Como a determinação da profundidade ideal da água (Gomes et al., 2020), o efeito da utilização de água salinizada (Fabregat et al., 2017) e a densidade de estocagem das larvas (Dias et al., 2016) nos primeiros dias de vida.

O fornecimento de alimento vivo é essencial para a sobrevivência de diversas espécies ornamentais, sendo o microcrustáceo Artemia sp. um dos alimentos vivos mais utilizados na larvicultura de peixes ornamentais por promover bons resultados zootécnicos, como os observados na produção de quinguio (Soares et al., 2000), acará- servero (Campelo et al., 2020), acará-bandeira (Pereira et al., 2016) e no Betta splendens (Couto et al., 2018).

A artêmia possui alto valor biológico, apresentando 57,26\% de proteína e 16,21\% de lipídeos em sua composição na fase de náuplios (Luna-Figueroa et al., 2010), porém, o seu fornecimento representa custo elevado devido a alta demanda no mercado e diminuição dos estoques naturais (Conceição et al., 2010). Tornando necessária a busca por alimentos vivos de menor custo que possam substituir total ou parcialmente o fornecimento de artêmia, como o microverme da aveia (Panagrellus redivivus).

O Panagrellus redivivus é um nematódeo de vida livre, cultivado em uma mistura de aveia e água, possuindo alto nível de proteína 44,22\% e 11,31\% de lipídeos. No entanto, sua utilização como alimento exclusivo se mostrou ineficiente na larvicultura do peixe anjo (Luna-Figueroa et al., 2010) e do Betta splendens (Couto et al., 2018), havendo a necessidade de avaliar sua utilização conjunta com outros alimentos vivos. 
Mediante o exposto, o presente trabalho foi desenvolvido com o objetivo de avaliar, por meio de índices de crescimento, desenvolvimento e sobrevivência, o fornecimento do microverme de forma exclusiva ou associado a artêmia e plâncton, como estratégia alimentar na larvicultura do peixe ornamental Betta splendens.

\section{Metodologia}

O presente estudo foi desenvolvido no Laboratório de Aquicultura do Centro de Ciências Humanas, Sociais e Agrárias (CCHSA) da Universidade Federal da Paraíba, situado no município de Bananeiras, Paraíba - Brasil. Foram utilizadas 450 larvas da espécie ornamental Betta splendens com três dias de vida, sendo submetidas ao período experimental até o $15^{\circ}$ dia de vida. Os exemplares foram obtidos a partir da desova de cinco casais, que ocorreram em um intervalo de 12 horas, promovendo a homogeneidade da idade das larvas.

O inicio do período experimental, 3 dias após eclosão, foi definido levando em consideração a funcionalidade do intestino e capacidade de ingestão de alimento. Segundo Silva et al. (2016) larvas da espécie Betta splendens apresentam trato digestivo aberto (boca e ânus), absorção total do vitelo e plena capacidade de consumir alimentos 74 horas após eclosão.

Ao $3^{\circ}$ dia de vida após eclosão, as larvas foram homogeneizadas, contadas e distribuídas em 15 unidades experimentais na proporção de 30 larvas por litro. Sendo a unidade experimental formada por aquário de vidro, com fundo verde para facilitar a visualização do alimento pelas larvas, capacidade para 2 litros, sendo abastecido com 1 litro de água. Antes da distribuição, foi recolhida uma amostra com 50 larvas para obtenção do comprimento total inicial (mm) com auxilio de paquímetro digital.

Foram avaliados três protocolos de alimentação com a utilização do microverme da aveia (Panagrellus redivivus), onde, no tratamento 1 foi fornecido microverme como único alimento; no tratamento 2 foram fornecidos microverme e artêmia (Artemia sp.); e no tratamento 3 foram fornecidos microverme e plâncton. Sendo o ensaio desenvolvido em um delineamento inteiramente casualizado com três tratamentos e cinco repetições.

Os alimentos vivos foram fornecidos duas vezes ao dia até saciedade aparente. O microverme foi obtido a partir de cultura desenvolvida com farinha de aveia misturada a amostra "start" inicial de cultura anterior, sendo um alimento vivo de baixo custo de produção. A artêmia recém-eclodida foi obtida através do processo de incubação dos cistos em água salgada e posterior coleta dos náuplios. O plâncton foi coletado diariamente, em viveiro escavado, com a utilização de uma rede coletora de plâncton. A retirada de resíduos nos aquários foi realizada diariamente com renovação parcial da água, uma hora antes do fornecimento dos alimentos vivos no período da manhã.

Os parâmetros físico-químicos da água: oxigênio dissolvido, $\mathrm{pH}$ e temperatura foram mensurados em intervalos de 48 horas durante o período experimental, com o auxílio de peagâmetro (HANNA®) e Oxímetro/termômetro (ICEL®).

Ao final do experimento, as larvas foram pesadas, insensibilizadas por imersão em gelo e posteriormente medidas para obtenção das variáveis: Comprimento final $(\mathrm{mm})$; ganho em comprimento $(\mathrm{mm})$; peso final $(\mathrm{g})$; taxa de desenvolvimento específico $=[(($ In comprimento total final - In comprimento total inicial $) \div$ tempo $) \times 100]$; taxa de uniformidade do lote para comprimento $=[($ número de peixes com comprimento $\pm 20 \%$ da média $) /$ número total de peixes por unidade experimental $)$ x100] e sobrevivência $=$ [(número final de peixes/número inicial de peixes) $x 100]$. As larvas foram fotografadas com câmera digital, por meio de lupa eletrônica com aumento de $2 \mathrm{x}$.

Os dados obtidos foram analisados pelo teste $\mathrm{F}$ e, em caso de diferenças significativas as médias dos tratamentos foram comparadas pelo teste de Tukey $(\mathrm{P}<0,05)$.

\section{Resultados e Discussão}

Não houve diferença entre tratamentos para o oxigênio dissolvido na água $(\mathrm{mg} / \mathrm{l})$, variando entre 8,15 e 8,94 (tabela 
1); e para a temperatura $\left(24,8^{\circ} \mathrm{C}\right.$ e $\left.24,9^{\circ} \mathrm{C}\right) . \mathrm{O} \mathrm{pH}$ foi menor $(7,30)$ para a água dos peixes alimentado com o protocolo microverme + artêmia. No entanto todas as variáveis observadas permaneceram dentro do aceitável para a espécie, visto que, Gomes et al. (2018) observaram bom desenvolvimento de Bettas splendens juvenis mantidos sob condições semelhantes de qualidade da água.

Tabela 1 - Qualidade da água na larvicultura de peixes Betta splendens submetidos a diferentes estratégias alimentares

\begin{tabular}{lllll}
\hline \multirow{2}{*}{ Qualidade da água } & \multicolumn{2}{l}{ Estratégia alimentar } & & \\
\cline { 2 - 4 } & Microverme & $\begin{array}{l}\text { Microverme } \\
\text { Artêmia }\end{array}$ & $+\begin{array}{l}\text { Microverme } \\
\text { Plâncton }\end{array}$ & \\
\hline $\mathrm{pH}$ & $8,02^{\mathrm{a}}$ & $7,30^{\mathrm{b}}$ & $8,14^{\mathrm{a}}$ & 2,8 \\
Oxigênio dissolvido $(\mathrm{mg} / \mathrm{l})$ & $8,89^{\mathrm{a}}$ & $8,15^{\mathrm{a}}$ & $8,94^{\mathrm{a}}$ & 6,65 \\
Temperatura $\left({ }^{\circ} \mathrm{C}\right)$ & $24,90^{\mathrm{a}}$ & $24,80^{\mathrm{a}}$ & $24,90^{\mathrm{a}}$ & 0,68 \\
\hline
\end{tabular}

*Médias na mesma linha seguidas de letras distintas diferem $(\mathrm{P}<0,05)$ pelo teste de Tukey. Fonte: Autores (2021).

As larvas submetidas ao protocolo de alimentação composto pelo fornecimento conjunto de microverme e náuplios de artêmia apresentaram o maior comprimento final (7,85mm). As larvas que foram alimentadas com microverme e plâncton obtiveram o segundo melhor comprimento final $(7,14 \mathrm{~mm})$ e o pior resultado $(6,38 \mathrm{~mm})$ foi obtido no tratamento que consistia no fornecimento de microverme como único alimento. Houve diferença $(\mathrm{p}<0,05)$ também para o ganho em comprimento, sendo maior para os animais que receberam a dieta microverme + artêmia (4,55 mm), seguido dos alimentados com microverme + plâncton $(3,80 \mathrm{~mm})$ e microverme $(3,08 \mathrm{~mm})$, respectivamente (Tabela 2; Figura 1).

Tabela 2 - Crescimento e peso de peixes Betta splendens submetidos a diferentes estratégias alimentares.

\begin{tabular}{lllc}
\hline \multirow{2}{*}{ Variáveis } & \multicolumn{3}{c}{ Estratégia alimentar } \\
\cline { 2 - 4 } & Microverme & $\begin{array}{c}\text { Microverme }+ \\
\text { Artêmia }\end{array}$ & Microverme + \\
Plâncton
\end{tabular}

*Médias na mesma linha seguidas de letras distintas diferem $(\mathrm{P}<0,05)$ pelo teste de Tukey. Fonte: Autores (2021). 
Figura 1 - Larvas Betta splendens submetidas a diferentes estratégias alimentar. A - Larva alimentada com microverme e artêmia $(7,85 \mathrm{~mm}$ de comprimento total - CT), B - Larva alimentada com microverme (6,38 mm CT) e C - Larva alimentada com microverme e plâncton (7,14 mm CT).

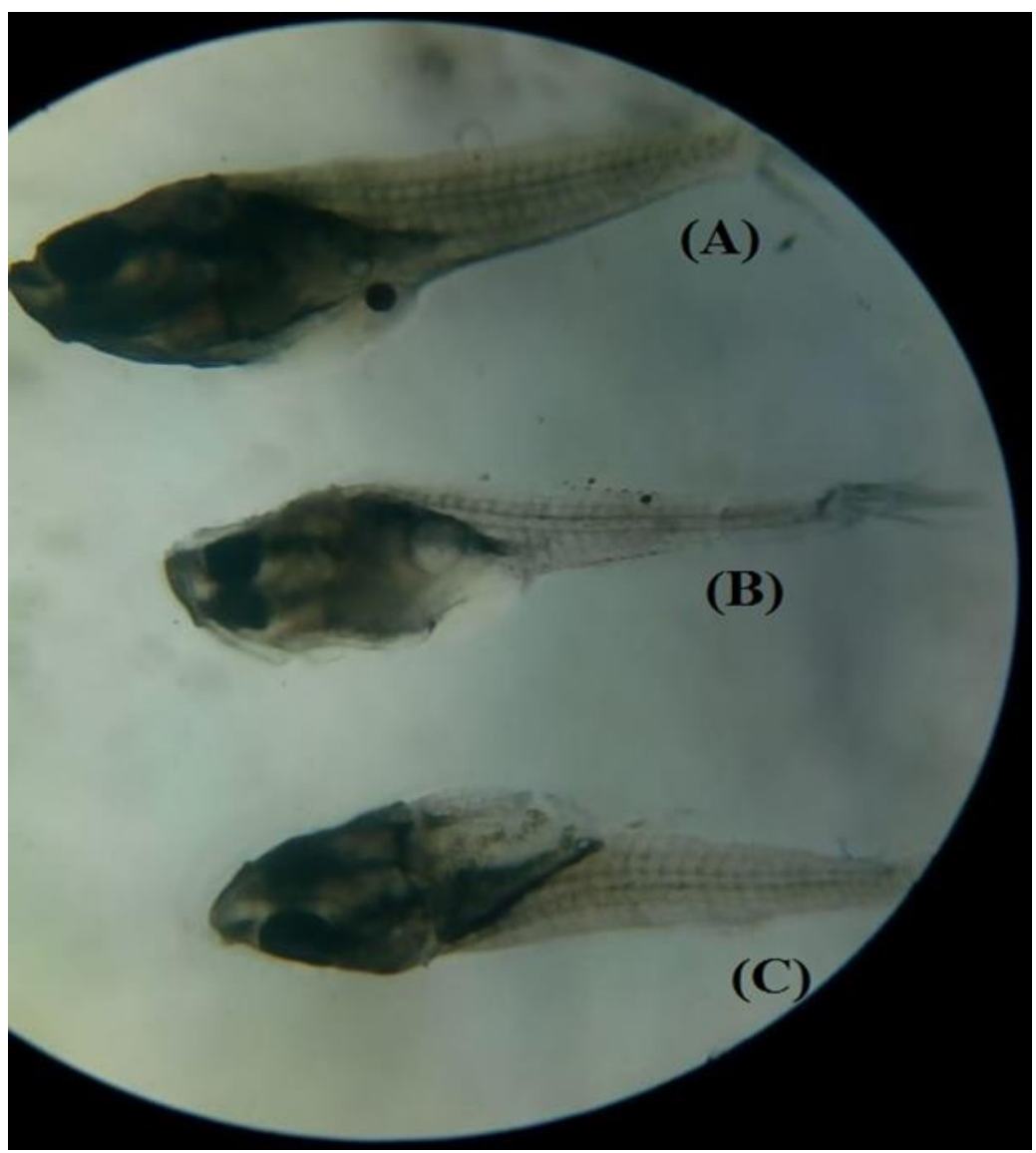

Fonte: Autores (2021)

As larvas submetidas ao protocolo de alimentação com o fornecimento conjunto de microverme e artêmia obtiveram o maior peso final, seguido das larvas submetidas ao protocolo alimentar com microverme e plâncton. As larvas alimentadas apenas com microverme apresentaram o menor peso final.

O fornecimento de artêmia é largamente utilizado na larvicultura de peixes ornamentais devido ao seu alto valor biológico. Campelo et al. (2020), ao avaliarem a utilização de artêmia, ração, infusório e uma combinação destes na alimentação do peixe ornamental acará servero, observaram maior comprimento total final e ganho em comprimento nos peixes alimentados com artêmia e com uma combinação de artêmia e ração extrusada. O maior ganho em peso também foi observado nos peixes que receberam artêmia como alimento exclusivo.

A necessidade de inclusão da artêmia durante a alimentação inicial de larvas Betta splendens observada no presente estudo pode ser atribuída ao alto valor nutricional e ao efeito desse alimento vivo no trato digestivo pelo estímulo ao seu desenvolvimento e à produção de enzimas digestivas que, consequentemente, melhoraram a digestão de nutrientes. Neste âmbito, Kolkovski (2001) sugere que o consumo de náuplios de artêmia por larvas de peixes pode favorecer a liberação de hormônios que influenciam na digestão, como a bombesina.

O fornecimento de alimento vivo durante a larvicultura de peixes é fundamental para o seu desenvolvimento e sobrevivência. Soares et al. (2000) ao avaliarem o fornecimento de artemia, plâncton e ração como alimentação inicial para larvas do peixe ornamental quinguio (Carassius auratus), observaram mortalidade de todos os exemplares que receberam 
ração como único alimento.

No entanto, a presença de fatores antinutricionais, características particulares do alimento vivo e a capacidade da larva de digerir um alimento específico devem ser analisadas. No presente estudo, o fornecimento do microverme da aveia como único alimento provocou os resultados mais baixos para comprimento final, ganho em comprimento e peso final. Estes resultados não podem ser atribuídos a falta de diversidade na alimentação, visto que, estudos testando dietas com fornecimento exclusivo de artêmia obtiveram resultados favoráveis. Como os relatados por Fosse et al. (2013), que ao avaliarem o fornecimento de alimento vivo (artêmia) e diferentes períodos de transição para o alimento inerte (coalimentação com artêmia + ração), observaram maior ganho em peso, peso final e comprimento final nas larvas Betta splendens alimentadas exclusivamente com náuplios de artêmia e nas larvas que foram submetidas ao maior período de coalimentação.

Ao avaliarem o desempenho de larvas Betta splendens alimentadas exclusivamente com artêmia, microverme ou regime alimentar alternado (artêmia + microverme), Couto et al. (2018) observaram maior ganho em peso para as larvas alimentadas com artêmias, seguida pelas larvas que consumiram artemia e microverme de forma conjunta, e os piores resultados foram obtidos nas larvas que consumiram microverme. A taxa de crescimento específico não diferiu entre os dois tratamentos, sendo inferior apenas nos exemplares que foram alimentados exclusivamente com microverme. Os autores atribuem estas diferenças à uma possível dificuldade das larvas em digerir nematoides de forma eficiente, no entanto, consideram o regime alimentar alternado (artêmia e microverme) o mais indicado para a larvicultura da espécie.

Segundo Luna-Figueroa et al. (2010), a porcentagem de proteína e lipídeos presentes na artêmia e no microverme é próxima, onde a artêmia possui 57,26\% de proteína e 16,21\% de lipídeos e o microverme apresenta 44,22\% de proteína e 11, $31 \%$ de lipídeos. No entanto, o fornecimento de microverme como alimento exclusivo apresentou resultados inferiores para peso final entre os alimentos vivos testados para larvas do peixe anjo (Pterophyllum scalare). Os autores atribuem este resultado a uma possível dificuldade na digestão da cutícula dos nematódeos, por ser composta por diferentes camadas.

A taxa de desenvolvimento específico $(\%)$ foi maior nas larvas submetidas ao protocolo alimentar microverme + artêmia. O segundo maior resultado foi encontrado nas larvas que consumiram plâncton e microvermes e o pior resultado foi encontrado nos exemplares que receberam dieta composta por microvermes (Tabela 3). A maior taxa de desenvolvimento específico observada nas larvas alimentadas com microverme e artêmia indica que estas obtiveram crescimento mais rápido durante o período de tempo analisado. Segundo Gomes et al. (2017), taxa de desenvolvimento elevado pode indicar menor tempo de produção e retorno financeiro mais rápido na piscicultura ornamental.

Tabela 3 - Desempenho zootécnico na larvicultura de peixes Betta splendens submetidos a diferentes estratégias alimentares.

\begin{tabular}{lllll}
\hline \multirow{2}{*}{ Variáveis } & \multicolumn{2}{l}{ Estratégia alimentar } & \\
\cline { 2 - 4 } & Microverme & $\begin{array}{l}\text { Microverme } \\
\text { Artêmia }\end{array}$ & Microverme + \\
Plâncton $(\%)$ & \\
\hline Taxa de desenvolvimento específico (\%) & $4,390^{\mathrm{c}}$ & $5,770^{\mathrm{a}}$ & $5,141^{\mathrm{b}}$ & 6,24 \\
Taxa de uniformidade (\%) & $69,828^{\mathrm{ab}}$ & $82,111^{\mathrm{a}}$ & $57,722^{\mathrm{b}}$ & 15,04 \\
Sobrevivência $(\%)$ & $54,167^{\mathrm{b}}$ & $84,000^{\mathrm{a}}$ & $51,250^{\mathrm{b}}$ & 9,55 \\
\hline
\end{tabular}

*Médias na mesma linha seguidas de letras distintas diferem $(\mathrm{P}<0,05)$ pelo teste de Tukey. Fonte: Autores $(2021)$.

A taxa de uniformidade do lote no tratamento que recebeu microverme não diferiu $(\mathrm{p}<0,05)$ dos demais tratamentos, no entanto, as larvas submetidas ao regime alimentar com microverme e artêmia apresentaram melhor taxa de uniformidade (\%) quando comparas ás que foram alimentadas com microverme e plâncton. 
Segundo Gonçalves Júnior, et al. (2014), o estabelecimento da hierarquia na alimentação e a manutenção de território próximo a um local de alimentação podem reduzir a taxa de uniformidade de um lote de peixes. No presente estudo, a ausência de diferença entre a taxa de uniformidade das larvas que receberam apenas microverme e os demais tratamentos pode ser atribuída a distribuição uniforme deste alimento no fundo dos aquários, observada após fornecimento, que pode ter favorecido uma disponibilidade homogênea de alimento, permitindo menor disputa entre indivíduos dominantes e não dominantes.

Takahashi et al. (2010), observaram que peixes ornamentais acará-bandeira alimentados com artêmia obtiveram melhores resultados na uniformidade do lote quando comparados aos alimentados com ração, atribuindo este resultado ao fato do alimento vivo ocupar toda a extensão do aquário e favorecer a oferta de alimento para todos os peixes.

A maior taxa de sobrevivência foi observada nos animais mantidos sobre a estratégia alimentar com fornecimento conjunto de microverme e artêmia, enquanto não houve diferença $(\mathrm{p}<0,05)$ para sobrevivência entre os demais tratamentos. A maior sobrevivência dos peixes pode ser atribuída a melhor condição corporal e possível aproveitamento eficiente de nutrientes da dieta composta por microverme e artêmia, pois, culminou também em maior peso, comprimento e taxa de desenvolvimento específico.

Larvas do peixe ornamental onívoro quinguio apresentaram sobrevivência mais alta quando foram alimentados com náuplios de artêmia ou alimentação conjunta de ração + artêmia durante os primeiros dias pós-eclosão. No entanto, as larvas que foram alimentadas com plâncton ou plâncton + ração obtiveram menor sobrevivência (Soares et al. 2000), corroborando com os resultados obtidos no presente trabalho, apesar das diferenças de hábito alimentar das espécies.

A estratégia alimentar preconizando o fornecimento de microverme da aveia de forma combinada aos náuplios de artêmia possibilitou melhores resultados na larvicultura do peixe ornamental Betta splendens, permitindo assim a incorporação de um ingrediente de baixo custo e fácil produção ao alimento vivo mais utilizando na nutrição de larvas de peixes.

Outro pronto crítico relacionado ao manejo alimentar durante a larvicultura dos peixes ornamentais é o período de transição entre o fornecimento de alimento vivo e alimento inerte. Este período pode variar de acordo com a espécie ornamental, sendo recomendada a transição após 10 dias para o acará-servero (Campelo et al., 2019) e após 20 dias para o acará-bandeira (Pereira el al., 2016). Como isso, recomenda-se que estudos sejam realizados com o intuito de definir a estratégia alimentar mais eficaz para transição do alimento vivo, avaliado no presente trabalho, para ração inerte durante a larvicultura do Betta splendens.

\section{Conclusão}

Nas condições do presente estudo, recomenda-se como estratégia alimentar o fornecimento de microverme da aveia e náuplos de artêmia de forma conjunta por promover os melhores índices de crescimento, desenvolvimento e sobrevivência na larvicultura inicial do peixe ornamental Betta splendens.

\section{Referências}

Campelo, D. A. V., Rodrigues, E. R., Dias, B. C. B., Pinheiro Junior, A. S., Santos, L. D., Brabo, M. F., Moura, L. B., \& Veras, G. C. (2020). Utilização de diferentes alimentos na larvicultura do peixe ornamental amazônico Acará Severo. Brazilian Journal of Development, 6 (3), 14035 -14049.

Campelo, D. A. V., Silva, I. C., Marques, M. H. C., Eiras, B. J. C. F., Brabo, M. F., De Moura, L. B., \& Veras, G. C. (2019). Estratégias alimentares na larvicultura do peixe ornamental amazônico acará-severo (Heros severus)(Heckel, 1840). Arquivo Brasileiro de Medicina Veterinária e Zootecnia, 71(5), $1601-1608$

Conceição, L.E.C., Yúfera, M., Makridis, P., Moraes, F., \& Dinis, M. T. (2010). Live feeds for early stages of fish rearing. Aquaculture Research. 4 (1), 613640 .

Couto, M. V. S. D., Sousa, N. D. C., Abe, H. A., Dias, J. A. R., Meneses, J. O., Paixao, P. E. G., Cunha, F. S., Ramos, F. M., Maria, A. N., Carneiro, P. C. F., $\&$ Fujimoto, R. Y. (2018). Effects of live feed containing Panagrellus redivivus and water depth on growth of Betta splendens larvae. Aquaculture Research. $48(8), 2671-2675$. 
Dias, J. A. R., Abe, H. A., Sousa, N. C., Ramos, F. M., Cordeiro, C. A. M., \& Fujimoto, R. Y. (2016). Efeito do sal (NaCl) e da densidade de estocagem na larvicultura de Betta splendens. Boletim do Instituto de Pesca, 42 (3), 719-726.

Fabregat, T. E. H. P., Wosniak, B., Takata, R., Miranda Filho, K.C., Fernandes, J. B. K.; \& Portella, M.C.(2017). Larvicultura do betta em água levemente salinizada. Boletim do Instituto de Pesca, 43 (2), 164-171.

Fosse, P. J., Mattos, D. C., Cardoso, L. D., Motta, J. H. S., Jasper, A. P. S., Radael, M. C., Andrade, D. R., \& Vidal Júnior, M. V. (2013). Estratégia de coalimentação na sobrevivência e no crescimento de larvas de Betta splendens durante a transição alimentar. Arquivo Brasileiro de Medicina Veterinária e Zootecnia. 65 (6), 1801-1807.

Gomes, V. D. S., Amâncio, A. L. L., Cavalcanti, C. R., \& Araújo, M. h. c. (2020). Efeito da profundidade da água na larvicultura do Betta splendens. Revista Científica Rural, 22 (2), $332-342$.

Gomes, V. D. S., Amâncio, A. L. L., Jordão Filho, J., Cavalcanti, C. R., Batista, J. M. M., \& Silva, J. H.V. (2018). Índices de desenvolvimento em juvenis Betta splendens alimentados com aditivos enzimáticos. Visão acadêmica, 19 (4), 46-54.

Gomes, V. D. S., Silva, J. H. V., Cavalcanti, C. R., Lima, M. C., Jordão Filho, J., \& Amâncio, A. L. L. (2017). Enzimas exógenas na alimentação do peixe guppy (Poecilia reticulata). Archives of Veterinary Science. 22 (3), 24-29.

Gonçalves Junior, P. L., Mendonça, P. P., Pereira, S. L., Matielo, M. D., \& Amorim, I. R. S. (2014). Densidade de estocagem durante a larvicultura do kinguio. Boletim do Instituto da Pesca, 40 (4), 597-604.

Kolkovski, S. (2001). Digestive enzimes in fish larvae and juveniles-implications and applications to formulated diets. Aquaculture, 200 (1), $181-201$.

Luna-Figueroa, J., Vargas, Z. T. J., \& Figueroa, T. J. (2010). Alimento vivo como alternativa en la dieta de larvas y juveniles de Pterophyllum scalare (Lichtenstein,1823). Avances en Investigación Agropecuaria, 14 (3), 63-72.

Pereira, S. L., GONÇALVES, L. P., Azevedo, R. V. D., Matielo, M. D., Selvatici, P. D. C., Amorim, I. R., \& Mendonça, P. P. (2016). Diferentes estratégias alimentares na larvicultura do acará-bandeira (Peterolophyllum scalare, Cichlidae). Acta Amazonica, 46 (1), 91-98.

Ramos, A., \& Gonçalves, D. (2019). Artificial selection for male winners in the Siamese fighting fish Betta splendens correlates with high female aggression. Frontiers in Zoology, 16 (34), $1-12$.

Silva, R. C., Valentin, F. N., Paes, M. C. F., Faustino, F., Giannecchini, L. G., Viadanna, P. H. O., Nascimento, N. F., Fernandes, J. B. K., \& Nakaghi, L. S. O. (2016). Development of the digestive tract in first feeding larvae of Betta splendens Regan, 1910. Journal of Applied Ichthyology, 32 (5), 840-847.

Soares, C. M., Hayashi, C., Gonçalves, G. S., \& Galdioli, E. M. (2000). Plâncton, Artemia sp, dieta artificial e suas combinações no desenvolvimento e sobrevivência do quinguio (Carassius auratus) durante a larvicultura. Acta Scientiarum. Biological Sciences, 2 (2), $383-388$.

Srikrishnan, R., Hirimuthugoda, N., \& Rajapakshe, W. (2017). Evaluation of growth performance and breeding habits of fighting fish (Betta splendens) under 3 diets and shelters. Journal of Survey in Fisheries Sciences, 3 (2). 50 - 65.

Takahashi, L. S., Silva, T. V., Fernandes, J. B. K., \& Mendonça, P. P. (2010). Efeito do tipo de alimento no desempenho produtivo de juvenis de acarábandeira (Pterophyllum scalare). Boletim do Instituto da Pesca, 36 (1), 1-8.

Zuanon, J. A. S., Salaro, A. L., Veras, G. C., \& Tavares, M. M. (2009). Tolerância aguda e crônica de adultos de beta, Betta splendens, à salinidade da água. Revista Brasileira de Zootecnia, 38 (11), 2106-2110. 